\title{
The ferromagnetic and antiferromagnetic phases in anion deficient $\mathrm{La}_{0.5-\mathrm{x}} \mathrm{Pr}_{\mathrm{x}} \mathrm{Ba}_{0.5} \mathrm{CoO}_{3-\delta}$ cobaltites
}

\author{
I. O. Troyanchuk, ${ }^{1, a)}$ D. V. Karpinsky, ${ }^{1,2}$ M. V. Bushinsky, ${ }^{1}$ V. Sikolenko, ${ }^{3,4}$ V. Efimov, ${ }^{4}$ \\ A. Cervellino, ${ }^{5}$ and B. Raveau ${ }^{6}$ \\ ${ }^{1}$ Scientific-Practical Materials Research Centre of NAS of Belarus, P. Brovka Street 19, BY-220072 Minsk, \\ Belarus \\ ${ }^{2}$ CICECO/Department of Ceramics and Glass Engineering, University of Aveiro, PT-3810-193 Aveiro, \\ Portugal \\ ${ }^{3}$ Karlsruhe Institute of Technology, DE-76131 Karlsruhe, Germany \\ ${ }^{4}$ Joint Institute for Nuclear Research, Dubna RU-141980, Russia \\ ${ }^{5}$ Swiss Light Source, Paul Scherrer Institute, CH-5232 Villigen, Switzerland \\ ${ }^{6}$ Laboratoire CRISMAT, UMR 6508 Associée au CNRS, ISMRA et Universite de Caen 6 , \\ Boulevard du Marechal Juin, 14050 Caen Cedex, France
}

(Received 5 April 2012; accepted 5 June 2012; published online 11 July 2012)

\begin{abstract}
Neutron powder diffraction studies of crystal and magnetic structures, magnetization and magnetotransport measurements have been performed for $\mathrm{La}_{0.5-\mathrm{x}} \mathrm{Pr}_{\mathrm{x}} \mathrm{Ba}_{0.5} \mathrm{CoO}_{3-\delta} \quad(\mathrm{x} \leq 0.375$, $\delta<0.25)$ cobaltites. It has been found that the compositions $(0 \leq \mathrm{x} \leq 0.35)$ are cubic $(P m 3 m)$ whereas $\mathrm{x}=0.375$ one is tetragonal $(P 4 / \mathrm{mmm})$ due to an ordering of rare-earth and barium ions, while the oxygen vacancies are disordered. The oxygen content decrease leads to transformation of the magnetic structure from ferromagnetic to the G-type antiferromagnetic one through the mixed two-phase magnetic state. The compositions with antiferromagnetic component show structural phase separation, strong increase of the unit cell volume upon cooling, and anomalous magnetization behavior. It is suggested that these phenomena are associated with stabilization of $\mathrm{Co}^{3+}$ ions in high/low spin state at low temperature. The high/low spin state corresponds antiferromagnetic phase, whereas in the ferromagnetic one, the $\mathrm{Co}^{3+}$ and $\mathrm{Co}^{4+}$ ions adopt intermediate spin state. (C) 2012 American Institute of Physics. [http://dx.doi.org/10.1063/1.4733953]
\end{abstract}

\section{INTRODUCTION}

Rare earth cobaltites $\mathrm{Ln}_{1-\mathrm{x}} \mathrm{A}_{\mathrm{x}} \mathrm{CoO}_{3}(\mathrm{Ln}=$ lanthanide, $\mathrm{A}=$ alkaline earth metal: $\mathrm{Ca}, \mathrm{Sr}$, or $\mathrm{Ba}$ ) with perovskite structure attract much interest as they exhibit a variety of unusual magnetic and transport properties. ${ }^{1-5}$ The Co ions in octahedral symmetry may have either high, intermediate, or low-spin state as the energies of the crystal-field splitting of both the Co $3 d$ states and the Hund's rule exchange energy are comparable. In the ground state at low temperature, $\mathrm{LaCoO}_{3}$ contains $\mathrm{Co}^{3+}$ ions with the low-spin electronic configuration $t_{2 g}^{6} e_{g}^{0}{ }^{1-3}$ Upon heating, the spin state of Co ions thermally activates to the intermediate state (IS, $t_{2 g}^{5} e_{g}^{1}$, $S=1$ ) or high-spin state (HS, $\left.t_{2 g}^{4} e_{g}^{2}, S=2\right){ }^{1-3}$

In the hole-doped cobaltites, $\mathrm{La}_{1-x} \mathrm{~A}_{\mathrm{x}} \mathrm{CoO}_{3}$, the additional $\mathrm{Co}^{4+}$ ion increases the complexity of the system as it can also be in the different spin states. Among doped cobaltites, the system $\mathrm{La}_{1-\mathrm{x}} \mathrm{Sr}_{\mathrm{x}} \mathrm{CoO}_{3}$ is the most extensively investigated. A spin glass behavior was reported for $0.01<\mathrm{x}$ $<0.18$ as well as a ferromagnetic long-range ordering that apparently coincides with concentration insulator-to-metal transition for $\mathrm{x} \approx 0.18 .{ }^{6}$ Similar metallic ferromagnetic state was observed in barium-doped cobaltites with barium content $\mathrm{x}>0.2 .^{7-9}$ However, concentrational diamagneticferromagnetic transition occurs through intermediate

\footnotetext{
a) Author to whom correspondence should be addressed. Electronic mail: troyan@physics.by.
}

insulating antiferromagnetic state. ${ }^{7}$ Moreover, lightly doped ferromagnetic state $(0.18 \leq \mathrm{x} \leq 0.22)$ seems to be insulating. ${ }^{7}$ The structural studies performed on the cubic oxygenstoichiometric perovskite $\mathrm{La}_{0.5} \mathrm{Ba}_{0.5} \mathrm{CoO}_{3}$ have revealed an onset of a long-range tetragonal phase accompanying a paraferromagnetic transition at $\mathrm{T}_{C} \approx 180 \mathrm{~K} \cdot .^{10,11}$ The tetragonal distortion has been discussed in terms of cooperative static Jahn-Teller distortions of the $\mathrm{CoO}_{6}$ octahedra. It was assumed that the Jahn-Teller effect is favored by the intermediate spinstate configuration of the $\mathrm{Co}^{3+}\left(d^{6}\right)$ and $\mathrm{Co}^{4+}\left(d^{5}\right)$ ions derived from the measured ferromagnetic moment $-1.9 \mu_{B}$ per cobalt ion. However, the Sr-doped ferromagnetic cobaltites have approximately the same magnetic moment value and do not exhibit any structural transition at the Curie point. $^{12,13}$ Moreover, the extended $\mathrm{x}$-ray absorption fine structure (EXAFS) and neutron diffraction studies do not reveal any appreciable local Jahn-Teller distortion in $\mathrm{La}_{1-\mathrm{x}} \mathrm{Sr}_{\mathrm{x}} \mathrm{CoO}_{3} .{ }^{14}$ The nature of the ferromagnetic state in cobaltites was a subject of debates for a long time. ${ }^{15-17}$ Three main mechanisms explaining magnetic properties of mixedvalence cobaltites were suggested: the superexchange model based on the localized electron interaction via oxygen ion, the Zener double exchange via charge transfer, and the itinerant electron ferromagnetism. ${ }^{15-17}$

Beside the alkaline earth doping, there is another way to manipulate the physical properties of the rare-earth cobaltites. In accordance with Refs. 18 and 19, the oxygen deficiency in $\mathrm{La}_{0.5} \mathrm{~A}_{0.5} \mathrm{CoO}_{3-\delta}(\mathrm{A}=\mathrm{Sr}, \mathrm{Ba})$ perovskites is 
accompanied by a strong decrease of magnetization and phase separation phenomenon. It was shown that the oxygen content in the $\mathrm{Ln}$ and $\mathrm{Ba}$ ordered perovskite systems $\mathrm{LnBa}-$ $\mathrm{Co}_{2} \mathrm{O}_{\mathrm{y}}$ can be varied in a wide range from $\mathrm{y}=4.5$ to $\mathrm{y}=6$ what leads to a change in the average oxidation state of the cobalt ions from $2+$ to $3.5+.^{20-22}$ The intermediate composition $\mathrm{LnBaCo}_{2}^{3+} \mathrm{O}_{5.5}$ with ordered oxygen vacancies shows very interesting properties, such as metal-insulator transition, antiferromagnetic-ferromagnetic transition, and the giant magnetoresistance effect. ${ }^{20-22}$ The Curie point of LaBa$\mathrm{Co}_{2} \mathrm{O}_{5.5}$ is the largest and reaches $326 \mathrm{~K} .{ }^{23}$ Several studies of the magnetic structure of $\mathrm{LnBaCo}_{2} \mathrm{O}_{5.5}$ layered perovskites have been performed with diverging results. ${ }^{24-31}$ According to Refs. 27 and 31, the magnetic structure is non-collinear in both antiferromagnetic and ferromagnetic phases. The $\mathrm{Co}^{3+}$ ions in octahedra adopt the low-spin state, whereas in pyramids, they are in the high-spin state. ${ }^{27,31}$ In the works, ${ }^{24,25}$ it was suggested that the magnetic structure can be described by collinear ferromagnetic model. The symmetry analysis was used to support the collinear model of the magnetic structure. ${ }^{24,25}$ However, the giant anisotropic magnetoresistance effect associated with antiferromagnetic-ferromagnetic transition and magnetic structure evolution in Fe-doped layered cobaltites seems to be better explained by non-collinear magnetic structure model. ${ }^{32}$ It worth to be noting that the antiferromagnet-ferromagnet transitions have been revealed in $\mathrm{Sr}_{3} \mathrm{YCo}_{4} \mathrm{O}_{10.5}$ layered perovskites also. ${ }^{33}$

Considering these reports, we have decided to investigate the effect of oxygen deficiency on structural and magnetic properties of $\mathrm{La}_{0.5-\mathrm{x}} \mathrm{Pr}_{\mathrm{x}} \mathrm{Ba}_{0.5} \mathrm{CoO}_{3-\delta}$ cobaltites with ordinary or layered perovskite structure where oxygen vacancies are disordered. In the present work, it is shown that oxygen deficiency in these cobaltites leads to a concentrational transition from ferromagnetic to pure antiferromagnetic phase through mixed state. It is remarkable that the phase separation is accompanied with strong increase of the unit cell volume and anomalous temperature dependence of magnetization.

\section{EXPERIMENTAL}

Ceramic samples of $\mathrm{La}_{0.5-\mathrm{x}} \mathrm{Pr}_{\mathrm{x}} \mathrm{Ba}_{0.5} \mathrm{CoO}_{3-\delta}$ compositions were prepared by a solid-state phase reaction method using high-purity $\mathrm{La}_{2} \mathrm{O}_{3}, \mathrm{Pr}_{6} \mathrm{O}_{11}, \mathrm{BaCO}_{3}$, and $\mathrm{CoO}$ taken in stoichiometric ratio and thoroughly mixed in a planetary ball mill (RETSCH PM-100). The synthesis was performed at $1200{ }^{\circ} \mathrm{C}$ for $10 \mathrm{~h}$ in air, followed by cooling at different constant rate (varied from $50^{\circ} \mathrm{C} / \mathrm{h}$ to $300^{\circ} \mathrm{C} / \mathrm{h}$ ) down to $300^{\circ} \mathrm{C}$. Some samples were annealed during $48 \mathrm{~h}$ at $650-800^{\circ} \mathrm{C}$ and quenched. The phase purity was checked using a diffractometer DRON-3M. Synchrotron powder diffraction (SPD) experiments were carried out at the swiss light source synchrotron of the Paul Scherrer Institute using MS beamline (wavelength $0.49 \AA$ ) The neutron powder diffraction (NPD) experiments were performed using a high-resolution diffractometer E9 at the Berlin Neutron Scattering Center (BENSC) of Helmholtz-Zentrum Berlin (HZB) and high intensity diffractometer DMC at the Laboratory for Neutron Scattering of Paul Scherrer Institut (PSI, Villigen). The neutron and $\mathrm{X}$-ray powder diffraction data were analyzed by Rietveld method using FULlPROF software package. ${ }^{34}$ Magnetic and magnetotransport properties up to $14 \mathrm{~T}$ were studied with a physical properties measurement system (Cryogenic Ltd.).

\section{RESULTS AND DISCUSSION}

\section{A. Crystal and magnetic structure}

The crystal structure of the $\mathrm{La}_{0.5-\mathrm{x}} \mathrm{Pr}_{\mathrm{x}} \mathrm{Ba}_{0.5} \mathrm{CoO}_{3-\delta}$ solid solutions has been analyzed using $\mathrm{x}$-ray, neutron, and synchrotron diffraction techniques. It has been found that the structure of the $\mathrm{La}_{0.5-\mathrm{x}} \mathrm{Pr}_{\mathrm{x}} \mathrm{Ba}_{0.5} \mathrm{CoO}_{3-\delta}$ solid solutions changes essentially with an increase of $\mathrm{Pr}$ ions content. The XRD data obtained at room temperature verify the cubic structure of the $\mathrm{La}_{0.5-\mathrm{x}} \mathrm{Pr}_{\mathrm{x}} \mathrm{Ba}_{0.5} \mathrm{CoO}_{3-\delta}$ solid solutions up to concentration $\mathrm{x}=0.35$; further increase of praseodymium concentration promotes stabilization of the tetragonal distortion of the compounds.

The neutron diffraction data for the $\mathrm{La}_{0.5} \mathrm{Ba}_{0.5} \mathrm{CoO}_{3-\delta}$ sample cooled at a rate of $300^{\circ} \mathrm{C} / \mathrm{h}$ from $1200^{\circ} \mathrm{C}$ have been recorded at temperatures of $300 \mathrm{~K}, 150 \mathrm{~K}, 80 \mathrm{~K}$, and $2 \mathrm{~K}$. Rietveld refinement performed for the $300 \mathrm{~K}$ NPD pattern assumes cubic symmetry of the compound $(P m \overline{3} m$ space group). The structural analysis performed by using the tetragonal space group $(P 4 / \mathrm{mmm})$, rhombohedral $(R \overline{3} \mathrm{c})$, and orthorhombic one (Pnma) did not result in any essential improvement of the reliability factors and cubic structure has been assumed as a more feasible one. The value of the oxygen occupation refined from the NPD data at $300 \mathrm{~K}$ is about 2.88 , the cubic symmetry supposes a random distribution of the oxygen vacancies as well as of $\mathrm{La}$ and $\mathrm{Ba}$ ions. The crystal structure has been additionally studied by synchrotron powder diffraction. In order to estimate the structural parameters in detail, the SPD patterns were recorded in the range from $5 \mathrm{~K}$ up to $300 \mathrm{~K}$ at the $5 \mathrm{~K}$ step. The refinement of the SPD pattern recorded at $300 \mathrm{~K}$ has confirmed the macroscopic cubic symmetry $(P m \overline{3} m)$ of the $\mathrm{La}_{0.5} \mathrm{Ba}_{0.5} \mathrm{CoO}_{2.88}$. However, close inspection of the SPD patterns has revealed a very small asymmetric broadening of the diffraction peaks with temperature decreasing. Similar behavior of the NPD and SPD diffraction peaks excludes possible instrumental faults and/or texturing effects that could lead to an asymmetry of the peaks. Analysis of the NPD and SPD patterns recorded at temperatures below $150 \mathrm{~K}$ clarifies a cause of the phase instability. The most probable reason for the peak asymmetry is a gradual appearance of a new phase with structural parameters close to those for the parent phase. The diffraction patterns recorded at low temperatures have uniquely confirmed the phase separation scenario (Fig. 1). The structure refinement using two-phase model with cubic unit cells for the diffraction patterns obtained for $\mathrm{T}<150 \mathrm{~K}$ strongly improves the reliability factors. The refined structural data have confirmed gradual extension of the new cubic phase with temperature decrease. The phase separation process is reversible and well reproduced under heating/cooling. A relatively small difference in the unit cell parameters for the both the cubic phases hampers a precise estimation of the structural phase ratio. The content of the phase with the smaller lattice parameter calculated from the refinement of 


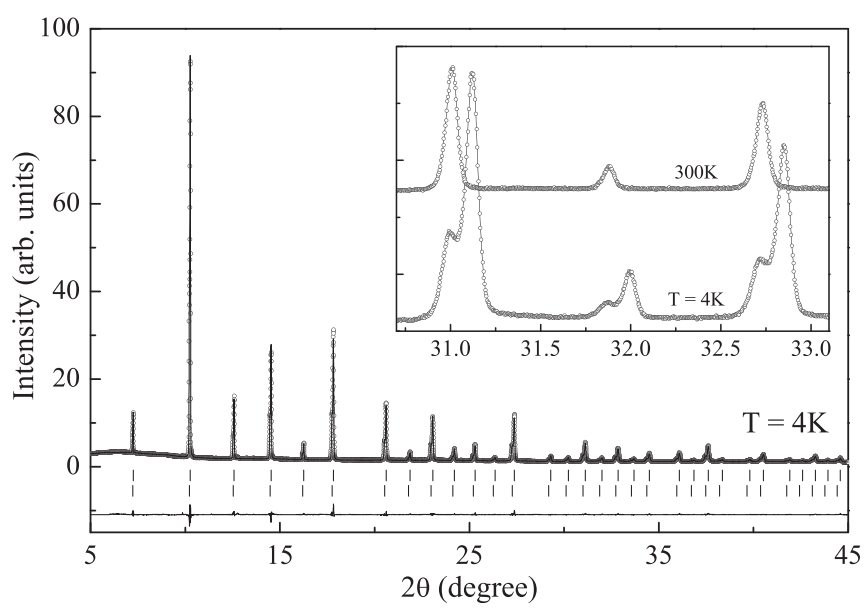

FIG. 1. The synchrotron diffraction spectra for the $\mathrm{La}_{0.5} \mathrm{Ba}_{0.5} \mathrm{CoO}_{3-\delta}$ compound at $4 \mathrm{~K}$. The observed and calculated profiles are noted by points and the line respectively, the bottom line represents their difference. The data are refined in $P m \overline{3} m$ space group for the both structural phases. The inset shows the magnified parts of the patterns at selected temperatures.

the NPD pattern recorded at $2 \mathrm{~K}$ is about $70 \%$ (the major phase). The structural data are given in Table I.

The rough estimation of the oxygen content performed for the NPD data taken at $2 \mathrm{~K}$ assumes the oxygen content for the phase with the larger lattice parameter to be smaller as compared with the major structural phase. However, the overlapping of the diffraction peaks hampers an accurate determination of the oxygen content for the both structural phases. One can suggest that in the minor phase, the cobalt ions have the oxidation state close to $3+$ and are located in the oxygen pyramids and octahedra, whereas in the major phase, the cobalt ions are placed predominantly in the oxygen octahedra and have a mixed $3+/ 4+$ oxidation state. It should be noted that stoichiometric $\mathrm{La}_{0.5} \mathrm{Ba}_{0.5} \mathrm{CoO}_{3}$ is a single phase down to the liquid helium temperature. ${ }^{10,11}$ So, the oxygen deficit is the important factor in the phase separation. It is worth emphasizing that phase segregation into electronrich (ferromagnetic) and electron-poor (antiferromagnetic) phases was earlier claimed for layered cobaltites $\mathrm{LnBaCo}_{2} \mathrm{O}_{6-\delta}\left(\mathrm{Ln}\right.$-lanthanide). ${ }^{35}$
The NPD data permit to clarify the magnetic structure of the $\mathrm{La}_{0.5} \mathrm{Ba}_{0.5} \mathrm{CoO}_{2.88}$. Taking into account the SPD data for $\mathrm{La}_{0.5} \mathrm{Ba}_{0.5} \mathrm{CoO}_{2.88}$, the additional peaks occurring only on NPD patterns below $150 \mathrm{~K}$ can be referred to magnetic neutron scattering (Fig. 2). The NPD patterns were well fitted assuming the coexistence of ferromagnetic and G-type antiferromagnetic structures. An additional contribution to the intensities of the diffraction peaks (100), (110), (210) testifies long-range ferromagnetic order within the compound, whereas the new magnetic peaks indexed as (111), (113), (313) in $2 a_{p} \times 2 a_{p} \times 2 a_{p}$ cubic metric assume antiferromagnetic ordering. Based on the structural data, the ferromagnetic contribution is attributed to the major structural phase, whereas G-type antiferromagnetic one is associated with the minor oxygen-poor phase with larger unit cell. The estimated magnetic moments are approximately $\sim 1.6 \mu_{B}$ for F-type phase and $\sim 2 \mu_{B}$ for G-type antiferromagnetic phase. The calculated moment for the ferromagnetic phase correlates with the published data for the stoichiometric $\mathrm{La}$ and $\mathrm{Ba}$ cation ordered $\mathrm{LaBaCo}_{2} \mathrm{O}_{6}\left(1.5 \mu_{B}\right)$ and cation disordered $\mathrm{La}_{0.5} \mathrm{Ba}_{0.5} \mathrm{CoO}_{3}\left(1.9 \mu_{B}\right)$ ferromagnetic phases. ${ }^{10,11}$

The NPD data obtained for the $\mathrm{La}_{0.5-\mathrm{x}} \mathrm{Pr}_{\mathrm{x}} \mathrm{Ba}_{0.5} \mathrm{CoO}_{3-\delta}$ compounds are in accordance with those received from $\mathrm{x}$-ray study at room temperature and clarify crystal structure evolution with temperature change. The NPD patterns for the $\mathrm{x}=0.25$ and 0.375 samples cooled at a rate of $300^{\circ} \mathrm{C} / \mathrm{h}$ from $1200^{\circ} \mathrm{C}$ were recorded in the temperature range from $\mathrm{T}=2 \mathrm{~K}$ up to $300 \mathrm{~K}$. Using the diffraction data, one can suggest that the crystal structure of the compound $\mathrm{x}=0.25$ is cubic in the whole measured temperature range. The phase separation phenomenon has not been observed. The crystal lattice symmetry of the $\mathrm{x}=0.375$ compound belongs to a tetragonal system. The NPD patterns of the $\mathrm{La}_{0.125} \mathrm{Pr}_{0.375} \mathrm{Ba}_{0.5} \mathrm{CoO}_{3-\delta}$ composition in the temperature range $2 \mathrm{~K}-300 \mathrm{~K}$ were well fitted using $P 4 / \mathrm{mmm}$ space group with $a_{p} \times a_{p} \times 2 a_{p}$ metric (Fig. 2). The doubling of the unit cell parameter along c-axis is a result of the ordering of the rare-earth and barium ions.

The Pr-doped solid solutions reveal exceptional behavior of the unit cell parameter with temperature change. For the

TABLE I. Structural parameters for the $\mathrm{La}_{0.25} \mathrm{Pr}_{0.25} \mathrm{Ba}_{0.5} \mathrm{CoO}_{2.79}, \mathrm{La}_{0.125} \mathrm{Pr}_{0.375} \mathrm{Ba}_{0.5} \mathrm{CoO}_{2.76}$, and $\mathrm{La}_{0.5} \mathrm{Ba}_{0.5} \mathrm{CoO}_{2.88}$ samples obtained by Rietveld refinement of the NPD patterns recorded at $2 \mathrm{~K}, 150 \mathrm{~K}$, and $300 \mathrm{~K}$.

\begin{tabular}{|c|c|c|c|c|c|c|c|c|c|c|}
\hline & \multicolumn{3}{|c|}{$\mathrm{La}_{0.25} \mathrm{Pr}_{0.25} \mathrm{Ba}_{0.5} \mathrm{CoO}_{2.79}$} & \multicolumn{3}{|c|}{$\mathrm{La}_{0.125} \mathrm{Pr}_{0.375} \mathrm{Ba}_{0.5} \mathrm{CoO}_{2.76}$} & \multicolumn{4}{|c|}{$\mathrm{La}_{0.5} \mathrm{Ba}_{0.5} \mathrm{CoO}_{2.88}$} \\
\hline & $\mathrm{T}=2 \mathrm{~K}$ & $\mathrm{~T}=150 \mathrm{~K}$ & $\mathrm{~T}=300 \mathrm{~K}$ & $\mathrm{~T}=2 \mathrm{~K}$ & $\mathrm{~T}=150 \mathrm{~K}$ & $\mathrm{~T}=300 \mathrm{~K}$ & $\mathrm{~T}=2 \mathrm{~K}$ & (2 phase) & $\mathrm{T}=150 \mathrm{~K}$ & $\mathrm{~T}=300 \mathrm{~K}$ \\
\hline $\mathrm{a}(\AA)$ & $3.8872(8)$ & $3.8824(4)$ & $3.8874(5)$ & $3.9114(5)$ & $3.9064(8)$ & $3.9132(3)$ & $3.8789(4)$ & $3.8944(5)$ & $3.8835(3)$ & $3.8900(6)$ \\
\hline$c(\AA)$ & & & & $7.6386(8)$ & $7.6223(7)$ & $7.6487(5)$ & & & & \\
\hline Vol (norm.) $\left(\AA^{3}\right)$ & 58.71 & 58.51 & 58.75 & 58.43 & 58.16 & 58.56 & 58.36 & 59.07 & 58.57 & 58.86 \\
\hline $\mathrm{Co}-\mathrm{O}(1)(\AA)$ & $1.9436(6)$ & $1.9412(5)$ & $1.9437(5)$ & $1.9675(5)$ & $1.9652(5)$ & $1.9674(4)$ & $1.9395(4)$ & $1.9473(3)$ & $1.9418(5)$ & $1.9450(4)$ \\
\hline $\mathrm{Co}-\mathrm{O}(2)(\AA)$ & & & & $1.9093(5)$ & $1.9118(7)$ & $1.9042(3)$ & & & & \\
\hline $\mathrm{Co}-\mathrm{O}(3)(\AA)$ & & & & $1.9099(7)$ & $1.8992(5)$ & $1.9201(4)$ & & & & \\
\hline Phase ratio & & & & & & & $70 \%$ & $30 \%$ & & \\
\hline $\begin{array}{l}\text { Magnetic } \\
\text { moment }\left(\mu_{B}\right)\end{array}$ & \pm 1.5 & \pm 0.6 & & \pm 1.6 & \pm 0.6 & & $1.6 \mathrm{~F}^{\mathrm{a}}$ & $\pm 2.1 \mathrm{AF}^{\mathrm{b}}$ & & \\
\hline$\chi^{2}$ & 2.63 & 2.79 & 2.27 & 2.97 & 2.83 & 2.64 & & & 4.31 & 4.63 \\
\hline
\end{tabular}

${ }^{\mathrm{a}} \mathrm{F}$, ferromagnetic.

${ }^{\mathrm{b}} \mathrm{AF}$, antiferromagnetic. 


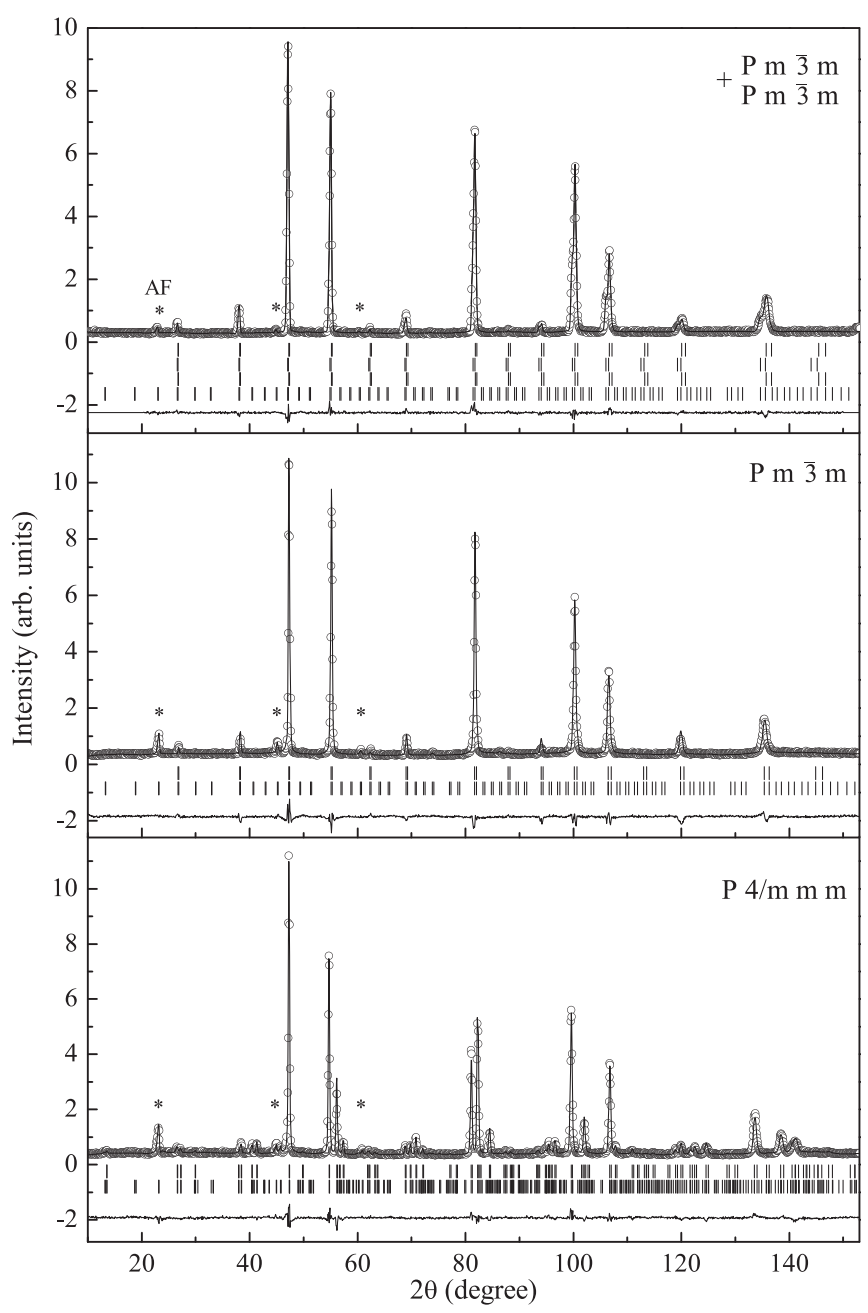

FIG. 2. The neutron powder diffraction patterns recorded at $2 \mathrm{~K}$ for $\mathrm{La}_{0.5} \mathrm{Ba}_{0.5}$ $\mathrm{CoO}_{2.88}$ (top panel), $\mathrm{La}_{0.25} \operatorname{Pr}_{0.25} \mathrm{Ba}_{0.5} \mathrm{CoO}_{2.79}$ (middle panel), $\mathrm{La}_{0.125} \operatorname{Pr}_{0.375}$ $\mathrm{Ba}_{0.5} \mathrm{CoO}_{2.76}$ (bottom panel) cooled at a rate of $300^{\circ} \mathrm{C} / \mathrm{h}$. The line and points refer to calculated and observed profiles, the bottom line represents their difference. For the $\mathrm{La}_{0.5} \mathrm{Ba}_{0.5} \mathrm{CoO}_{3-\delta}$ compound, two upper rows of the ticks ascribe structural phases, two bottom ones denote ferromagnetic and antiferromagnetic reflections corresponding to major and minor structural phases, respectively. For the Pr-doped compounds, the bottom ticks denote G-type antiferromagnetic structure, and antiferromagnetic reflections are marked by asterisks.

both $\mathrm{x}=0.25$ and 0.375 compounds, the unit cell parameter decreases with temperature reduction from room temperature down to $170 \mathrm{~K}$ where magnetic order develops. Whereas near the temperature of magnetic ordering, an unexpected increase of the lattice parameters is observed. The unit cell parameters estimated for the $\mathrm{La}_{0.5-\mathrm{x}} \mathrm{Pr}_{\mathrm{x}} \mathrm{Ba}_{0.5} \mathrm{CoO}_{3-\delta}$ compounds at $2 \mathrm{~K}$ are close to those observed at room temperature (Table I).

The occupation of the oxygen sites calculated from the NPD refinement for the $\mathrm{x}=0.25,0.375$ compounds assumes the oxygen deficit of $7 \%(\delta=0.21)$ and $8 \%(\delta=0.24)$, respectively. The estimated value of the oxygen deficit for $\mathrm{x}=0.375$ supposes approximately equal amounts of the oxygen octahedra and pyramids as well as $3+$ dominant oxidation state of the Co ions. Using the NPD data for $\mathrm{La}_{0.125} \mathrm{Pr}_{0.375} \mathrm{Ba}_{0.5} \mathrm{CoO}_{3-\delta}$, one can suppose the oxygen deficit to be mainly caused by vacancies in the $\mathrm{O}(2)$ oxygen sites. As oxygen vacancies are distributed randomly within the structure, the superexchange magnetic interactions are strongly frustrated. The low temperature neutron diffraction

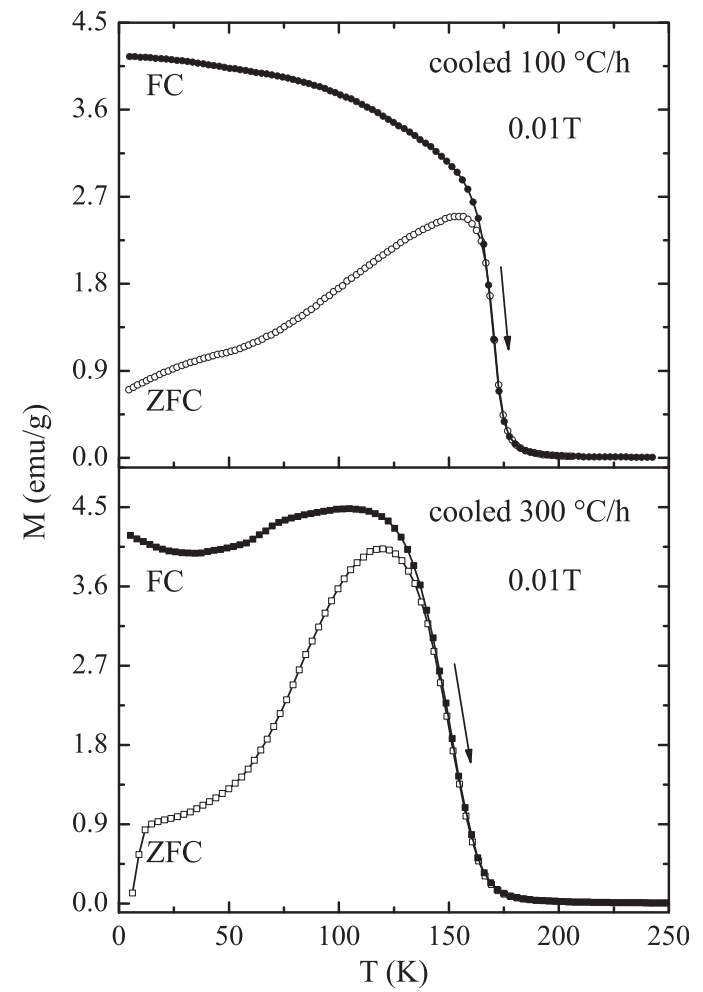

FIG. 3. Temperature dependencies of magnetization for $\mathrm{La}_{0.5} \mathrm{Ba}_{0.5} \mathrm{CoO}_{3-\delta}$ samples prepared under different thermal treatments. The arrows indicate direction of the temperature changing.

data assume dominant antiferromagnetic interactions within the both compounds. The NPD patterns below $170 \mathrm{~K}$ were well fitted assuming G-type antiferromagnetic structure of the compounds $\mathrm{x}=0.25,0.375$. The magnetic moments per cobalt ion for the compounds estimated from NPD study (see Table I) are much lower than those expected for high or even intermediate spin states. So, we can suppose different spin states for cobalt ions in the octahedra and the pyramids. In accordance with ${ }^{30,31}$ the cobalt ions in octahedra adopt a low spin state whereas in pyramids the high-spin state is expected. Alternatively, one can assume the presence of the spin-glass fraction which does not contribute to coherent neutron scattering. On the basis of intensities of the magnetic peaks the preferred orientation of the magnetic moment along $\mathrm{z}$-axis of the lattice was estimated for the compound $\mathrm{x}=0.375$ (see Table I).

In a more detailed consideration of the structural parameters for the $\mathrm{La}_{0.125} \mathrm{Pr}_{0.375} \mathrm{Ba}_{0.5} \mathrm{CoO}_{3-\delta}$, one can expect that the change of the unit cell volume is mainly caused by variation in $c$-parameter of the lattice. The increase of the $c$ parameter below the magnetic transition temperature is a result of shortening of the $\mathrm{Co}-\mathrm{O}(2)$ bond length and elongation of $\mathrm{Co}-\mathrm{O}(3)$ length, where $\mathrm{O}(3)$ and $\mathrm{O}(2)$ are apical oxygen ions. We suggest the most probable reason of the unusual expansion of the unit cell in the antiferromagnetic state to be stabilization of the $\mathrm{Co}^{3+}$ ions in the high-spin state (pyramids) and low-spin state (octahedra). Within ferromagnetic phase, both $\mathrm{Co}^{3+}\left(t_{2 g}^{5} e_{g}^{1}\right)$ and $\mathrm{Co}^{4+}\left(t_{2 g}^{4} e_{g}^{1}\right)$ adopt intermediate spin state. ${ }^{10}$ Alternatively, one can assume that there is a cooperative orbital ordering in the antiferromagnetically ordered phase as it was claimed for stoichiometric 
ferromagnetic $\mathrm{La}_{0.5} \mathrm{Ba}_{0.5} \mathrm{CoO}_{3} \cdot{ }^{10,11}$ However, an orbital ordering in ferromagnetic $\mathrm{La}_{0.5} \mathrm{Ba}_{0.5} \mathrm{CoO}_{3}$ does not lead to anomalous thermal expansion. ${ }^{10,11}$ It is worth stressing that layered perovskites $\mathrm{LnBaCo}_{2} \mathrm{O}_{5.5}$ do not exhibit unusual thermal expansion associated with magnetic ordering. ${ }^{23}$ So, one can conclude that oxygen vacancies disorder as well as large unit cell volume of $\mathrm{La}_{1-\mathrm{x}} \mathrm{Pr}_{\mathrm{x}} \mathrm{Ba}_{0.5} \mathrm{CoO}_{3-\delta}$ solid solutions are important factors apparently responsible for anomalous thermal behavior. Based on the structural data of the (La,Pr)-based cobaltites, we suggest the structural phase separation and abnormal thermal expansion of the unit cell volume peculiar for the antiferromagnetic minor phase of the $\mathrm{La}_{0.5} \mathrm{Ba}_{0.5} \mathrm{CoO}_{3-\delta}$ sample, to be associated with stabilization of the $\mathrm{Co}^{3+}$ ions in the high/low-spin state also. One can assume that at high temperature, the intermediate spin state is more stable.

\section{B. Magnetic properties}

The temperature dependencies of the magnetization $\mathrm{M}(\mathrm{T})$ of two representative samples of $\mathrm{La}_{0.5} \mathrm{Ba}_{0.5} \mathrm{CoO}_{3-\delta}$ with different oxygen content (oxygen content decreases with increase of cooling rate) are shown in Fig. 3. The zero field cooling (ZFC) and field cooling (FC) magnetizations for the compound with larger oxygen content (cooling at $100{ }^{\circ} \mathrm{C} /$ h) are similar to those for stoichiometric $\mathrm{La}_{0.5} \mathrm{Ba}_{0.5} \mathrm{CoO}_{3}$; however, the Curie point for stoichiometric compound is slightly higher. ${ }^{10,11}$ Decrease of the oxygen content (cooling $300^{\circ} \mathrm{C} / \mathrm{h}$ ) leads to a slight lowering of the Curie point and anomalous FC magnetization behavior. The onset of magnetic ordering starts at $170 \mathrm{~K}$ and shifts to a higher temperature as magnetic field increases. The magnetic moments estimated from $\mathrm{M}(\mathrm{H})$ curves (Fig. 4 ) are close to $1.75 \mu_{B} / \mathrm{Co}$ and $1 \mu_{B} /$ Co for the samples cooled at a rate of $100^{\circ} \mathrm{C} / \mathrm{h}$ and $300^{\circ} \mathrm{C} / \mathrm{h}$, respectively. The sample quenched from $700^{\circ} \mathrm{C}$ did not show any remanent magnetization.

The slowly cooled Pr-doped samples exhibit ferromagnetic-like behavior with Curie points close to $170 \mathrm{~K}$. However, the sample $\mathrm{La}_{0.25} \mathrm{Pr}_{0.25} \mathrm{Ba}_{0.5} \mathrm{CoO}_{3-\delta}$ fast cooled at a rate of $300^{\circ} \mathrm{C} / \mathrm{h}$ exhibit anomalous increase in magnetization with temperature increasing (Fig. 5). The magnetization starts

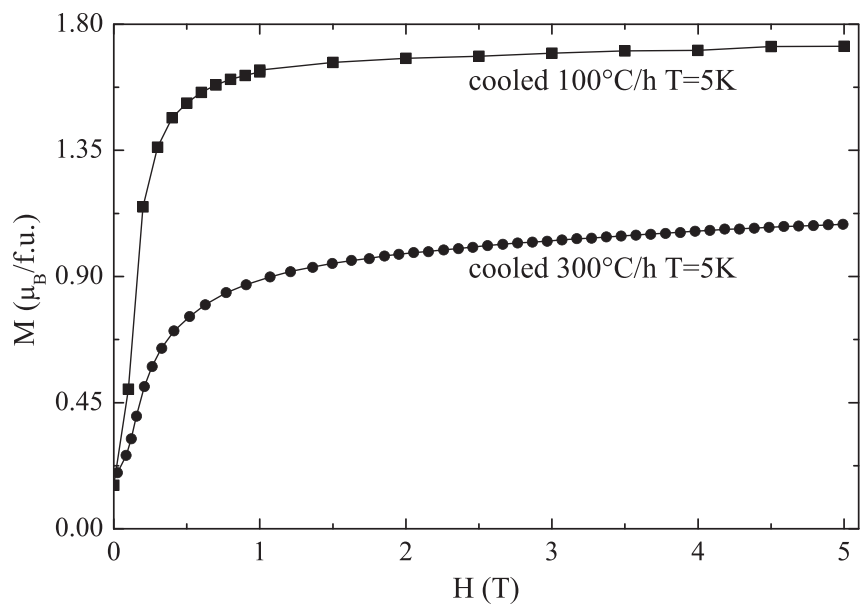

FIG. 4. Field dependencies of magnetization for $\mathrm{La}_{0.5} \mathrm{Ba}_{0.5} \mathrm{CoO}_{3-\delta}$ samples prepared under different thermal treatments.

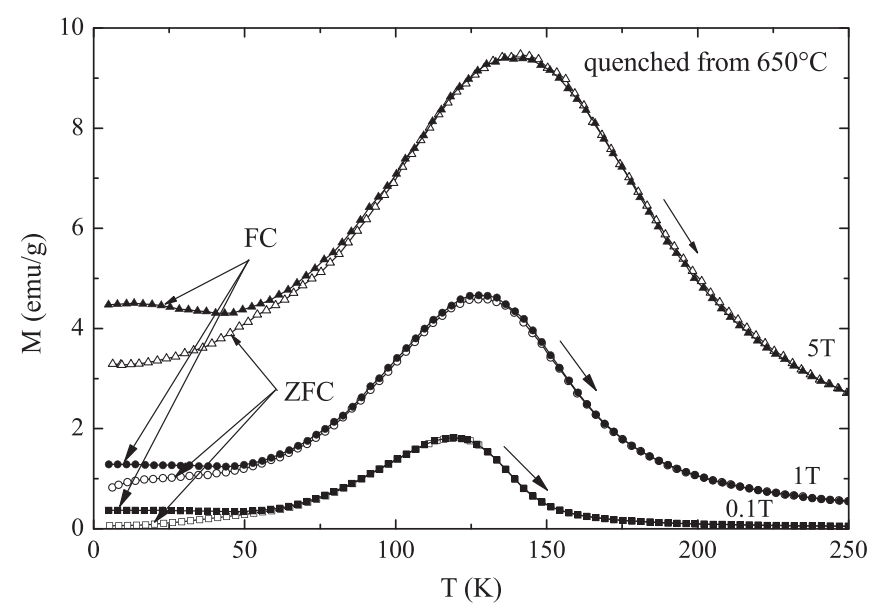

FIG. 5. Temperature dependencies of magnetizations for $\mathrm{La}_{0.25} \mathrm{Pr}_{0.25} \mathrm{Ba}_{0.5} \mathrm{CoO}_{3-\delta}$ samples prepared under different conditions.

to develop below $150 \mathrm{~K}$ in a field of $0.1 \mathrm{~T}$. The external magnetic field modifies the temperature dependence of the magnetization. The maximum of the magnetization in the field of $0.1 \mathrm{~T}$ was observed at $120 \mathrm{~K}$, whereas the field of $5 \mathrm{~T}$ shifts the maximum up to $150 \mathrm{~K}$. The $\mathrm{ZFC}$ and $\mathrm{FC}$ magnetizations dependencies measured in a large field of $5 \mathrm{~T}$, do not coincide below $70 \mathrm{~K}$, thus, indicating a huge magnetic anisotropy at low temperature. There is no appreciable temperature hysteresis between results of magnetization measurements carried out on warming and on cooling. The isothermal $\mathrm{M}(\mathrm{H})$ dependencies of magnetization for this sample are shown in Fig. 6. The spontaneous magnetization at $5 \mathrm{~K}$ is very small (about $0.5 \mathrm{emu} / \mathrm{g}$ ). The magnetic hysteresis practically disappears as temperature rises up to $70 \mathrm{~K}$, where anomalous enhancement of the magnetization starts to develop. The absent of the remanent magnetization indicates superparamagnetic state. The maximum of the magnetization derived from the $\mathrm{M}(\mathrm{H})$ dependencies was observed at $150 \mathrm{~K}$ similar to $\mathrm{M}(\mathrm{T})$ data. At $200 \mathrm{~K}$, the sample is in a paramagnetic state, however, the magnetization is much larger than that at $5 \mathrm{~K}$ in the field of $14 \mathrm{~T}$. So, we can conclude that at high temperature, the ferromagnetic interactions are dominant, whereas at low temperature, the antiferromagnetic interactions are prevailing. This behavior of the magnetization resembles $\mathrm{M}(\mathrm{T})$ dependence

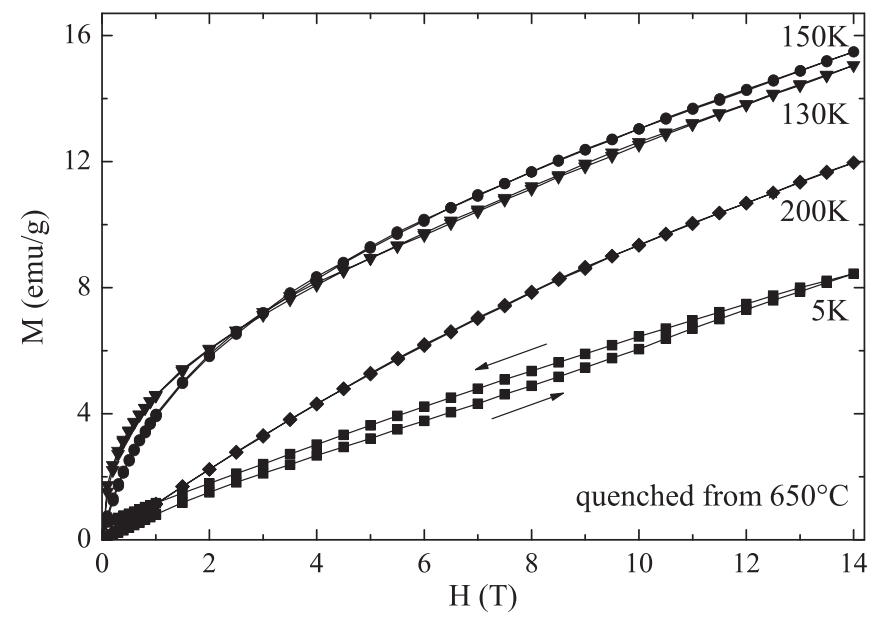

FIG. 6. Field dependencies of magnetization for $\mathrm{La}_{0.25} \operatorname{Pr}_{0.25} \mathrm{Ba}_{0.5} \mathrm{CoO}_{3-\delta}$ sample quenched from $650^{\circ} \mathrm{C}$. 
for $\mathrm{LnBaCo}_{2} \mathrm{O}_{5.5}$ oxygen ordered layered perovskites. These compounds exhibit antiferromagnet-"ferromagnet" transition as temperature rises. ${ }^{20-22}$ However for $\mathrm{LnBaCo}_{2} \mathrm{O}_{5.5}$ compounds, increasing of an external magnetic field shifts antiferromagnet-ferromagnet transition to low temperature.

Similar maximum of the magnetization was observed in $\mathrm{La}_{0.125} \mathrm{Pr}_{0.375} \mathrm{Ba}_{0.5} \mathrm{CoO}_{2.76}$ (taking into account the effect of ordering of the rare-earth and the barium ions, the chemical formula can be written as $\mathrm{La}_{0.25} \mathrm{Pr}_{0.75} \mathrm{BaCo}_{2} \mathrm{O}_{5.52}$ ) around $\mathrm{T}=130 \mathrm{~K}$ (Fig. 7). However, the magnitude of the magnetization is much lower than that for $\mathrm{La}_{0.25} \mathrm{Pr}_{0.25} \mathrm{Ba}_{0.5} \mathrm{CoO}_{2.79}$ with an ordinary perovskite structure. ZFC and FC magnetizations measured in the field of $0.01 \mathrm{~T}$ exhibit anomalous behavior near $50 \mathrm{~K}$ (Fig. 7, the upper panel), thus indicating another transition in the magnetically ordered phase. Most probably this transition is associated with a strong blocking of the superparamagnetic clusters due to interactions with antiferromagnetic phase. It worth emphasizing that similar anomalous magnetization behavior was observed for the sample $\mathrm{x}=0.35$ with ordinary perovskite structure.

The oxygen content estimated from the NPD data for the $\mathrm{La}_{0.25} \mathrm{Pr}_{0.25} \mathrm{Ba}_{0.5} \mathrm{CoO}_{2.79}$ is close to that for doublelayered perovskite $\mathrm{LaBaCo}_{2} \mathrm{O}_{5.5} \cdot{ }^{23}$ In the latter compound, $\mathrm{La}$ and $\mathrm{Ba}$ ions are ordered forming alternative layers with only one type of ions, and the oxygen vacancies are ordered as well. In accordance with the NPD study, ${ }^{23}$ the magnetic

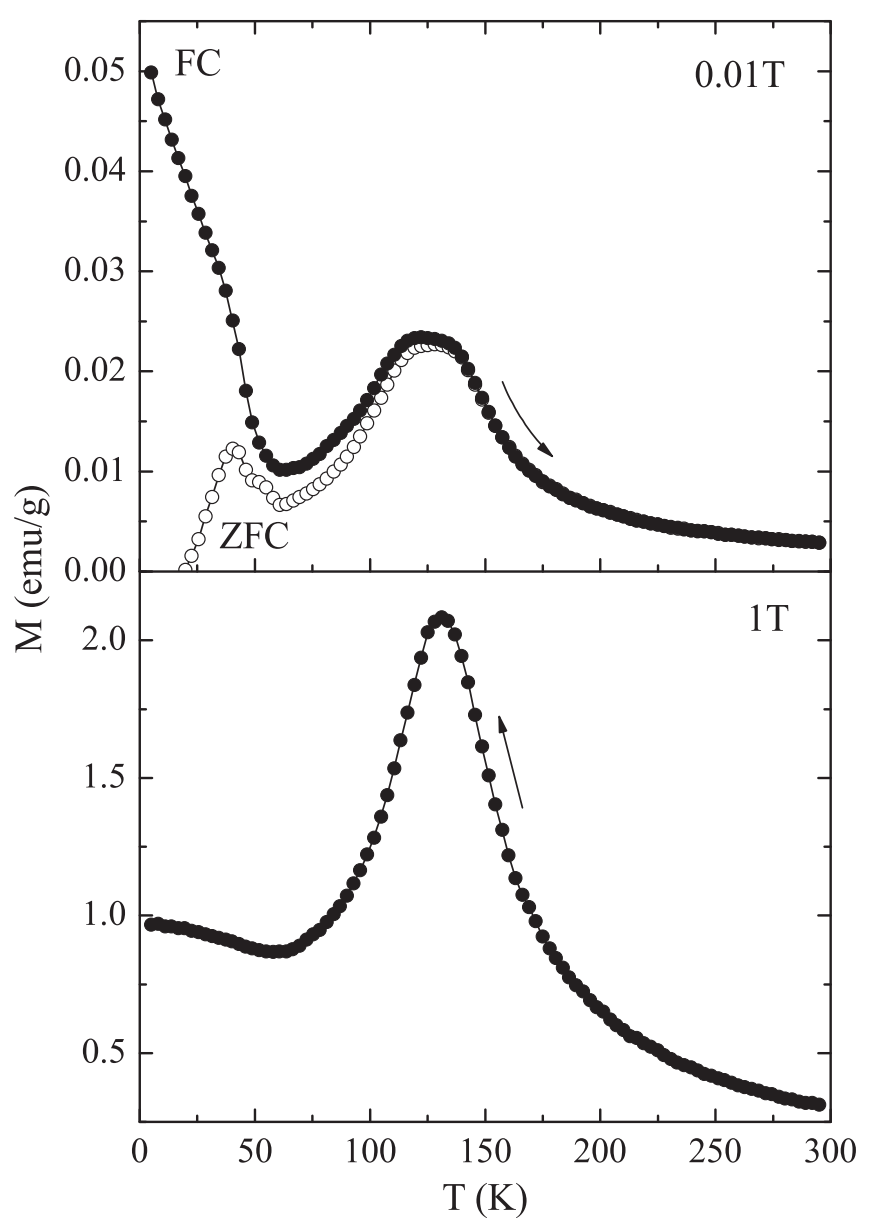

FIG. 7. Temperature dependencies of magnetization for $\mathrm{La}_{0.25} \mathrm{Pr}_{0.75} \mathrm{Ba}$ $\mathrm{Co}_{2} \mathrm{O}_{5.52}$ cooled at a rate of $300^{\circ} \mathrm{C} / \mathrm{h}$. structure is antiferromagnetic of G-type similar to that of disordered $\mathrm{La}_{0.25} \mathrm{Pr}_{0.25} \mathrm{Ba}_{0.5} \mathrm{CoO}_{3-\delta}$. However, the Neel point of the ordered phase is twice larger than that for the disordered phase. The higher Neel point of the ordered phase is associated with optimal geometry of negative and positive magnetic interactions. Both $\mathrm{La}_{0.25} \mathrm{Pr}_{0.25} \mathrm{Ba}_{0.5} \mathrm{CoO}_{2.79}$ and $\mathrm{LaBaCo}_{2} \mathrm{O}_{5.5}$ contain approximately one half of $\mathrm{Co}$ ions in $\mathrm{CoO}_{5}$ pyramids and other in $\mathrm{CoO}_{6}$ octahedra. The electronic configuration of $\mathrm{Co}$ ions located in the pyramids agrees with the high-spin state, whereas for Co ions in the octahedral surroundings, the low-spin state seems to be dominant. ${ }^{31,32}$ The calculated magnetic moment from NPD for the antiferromagnetic $\mathrm{La}_{0.25} \mathrm{Pr}_{0.25} \mathrm{Ba}_{0.5} \mathrm{CoO}_{2.79}$ phase is in agreement with this assumption. An expected magnetic moment value of the $\mathrm{Co}^{3+}$ (HS) ion is close to $3 \mu_{B}$ (Refs. 30 and 31), whereas the observed moment is $1.5 \mu_{B}$, thus suggesting much smaller magnetic moment in the octahedra. In the layered perovskites every $\mathrm{Co}^{3+}$ ion in the high-spin state interacts antiferromagnetically with three $\mathrm{Co}^{3+}$ (HS) ions and ferromagnetically with one $\mathrm{Co}^{3+}$ (HS) ion in the absence of a common oxygen bridge. ${ }^{32}$ There are only two $\mathrm{Co}^{3+}$ (LS) nearest neighbors. Another situation is realized in the disordered $\mathrm{La}_{0.25} \mathrm{Pr}_{0.25} \mathrm{Ba}_{0.5} \mathrm{CoO}_{2.79}$, where $\mathrm{Co}^{3+}$ (HS) ions interact only with three $\mathrm{Co}^{3+}$ (HS) ions on the average, and the positive and negative interactions are equally probable. In this case, there are three $\mathrm{Co}^{3+}$ (LS) nearest neighbors on the average. A random distribution and larger concentration of the $\mathrm{Co}^{3+}$ nearest neighbors in the low spin state favor the much lower Neel point in comparison with the layered cobaltites with ordered oxygen vacancies.

Let us consider a possible origin of the anomalous magnetization behavior observed in $\mathrm{La}_{0.25} \mathrm{Pr}_{0.25} \mathrm{Ba}_{0.5} \mathrm{CoO}_{2.79}$ and $\mathrm{La}_{0.125} \mathrm{Pr}_{0.375} \mathrm{Ba}_{0.5} \mathrm{CoO}_{2.76}$ compounds with the dominant antiferromagnetic structure. The small ferromagnetic component may be a result of the ferrimagnetic or non-collinear ferromagnetic structure similar to that suggested for doublelayered perovskites. ${ }^{24,31,32}$ However in $\mathrm{La}_{0.25} \mathrm{Pr}_{0.25} \mathrm{Ba}_{0.5-}$ $\mathrm{CoO}_{2.79}$, the oxygen vacancies as well as rare-earth and barium ions are disordered and there is only one structural site for cobalt ions, whereas four different structural sites are necessary for realization of the ferrimagnetic structure. ${ }^{24}$ The stabilization of the non-collinear magnetic structure does not satisfy the symmetry criteria. So one can suggest that ferromagnetic component has similar nature for all anion deficient compounds and corresponds to the dominant ferromagnetic interactions in some parts of the samples. Apparently at low temperature, the magnetic moments of the clusters are blocked due to interactions with dominant antiferromagnetic phase. The huge magnetic anisotropy developing in $\mathrm{La}_{0.25} \mathrm{Pr}_{0.25} \mathrm{Ba}_{0.5} \mathrm{CoO}_{3-\delta}$ at low temperature may be a result of the magnetic frustrations at the boundary between the pure antiferromagnetic phase and ferromagnetic clusters which coexist at low temperatures.

\section{Magnetotransport properties}

A relatively large negative magnetoresistance is observed in $\mathrm{La}_{0.5} \mathrm{Ba}_{0.5} \mathrm{CoO}_{3-\delta}$ cooled at a rate of $10{ }^{\circ} \mathrm{C} / \mathrm{h}$. Fig. 8 shows the temperature variations of electrical resistivity measured at 


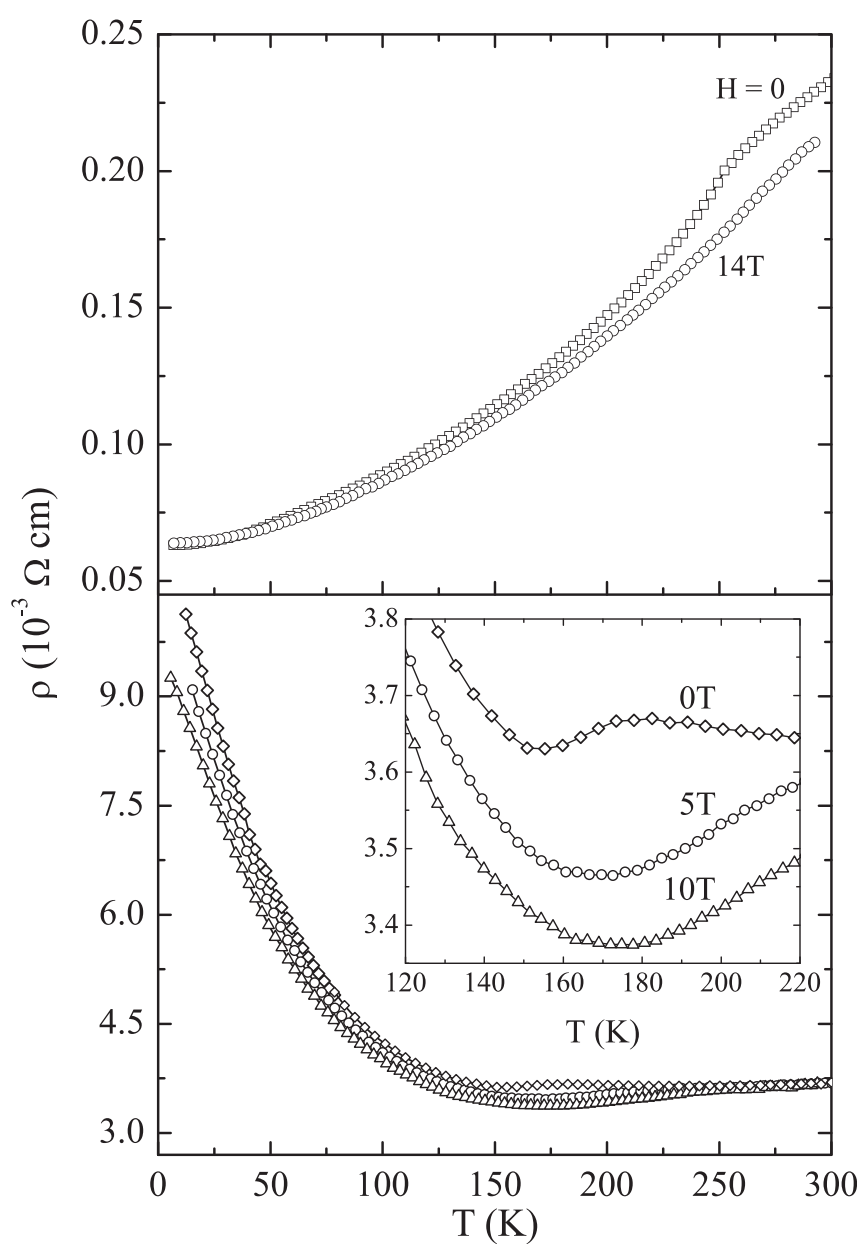

FIG. 8. The resistivity of ferromagnetic $\mathrm{La}_{0.5} \mathrm{Sr}_{0.5} \mathrm{CoO}_{3-\delta}$ (top panel) and $\mathrm{La}_{0.5} \mathrm{Ba}_{0.5} \mathrm{CoO}_{3-\delta}$ (bottom panel) cooled at a rate of $100^{\circ} \mathrm{C} / \mathrm{h}$ versus temperature. The inset shows the resistivity behavior near the Curie point.

different external magnetic fields for $\mathrm{La}_{0.5} \mathrm{Sr}_{0.5} \mathrm{CoO}_{3-\delta}$ (upper panel) and $\mathrm{La}_{0.5} \mathrm{Ba}_{0.5} \mathrm{CoO}_{3-\delta}$ (lower panel). Both the samples are ferromagnets and were cooled at a rate of $100^{\circ} \mathrm{C} / \mathrm{h}$. The resistivity of $\mathrm{La}_{0.5} \mathrm{Sr}_{0.5} \mathrm{CoO}_{3-\delta}$ exhibits the metallic behavior within the whole measured temperature range from 5 to $300 \mathrm{~K}$. The non-pronounced anomaly in the $\rho(\mathrm{T})$ dependence was observed near the Curie point at the absence of the external magnetic field. The magnetoresistance is not revealed at low temperatures but slightly appeared around $\mathrm{T}_{C}$, as shown in Fig. 8. The temperature variation of resistivity for $\mathrm{La}_{0.5} \mathrm{Ba}_{0.5} \mathrm{CoO}_{3-\delta}$ is dramatically different. The metallic behavior of the resistivity has been observed only in the narrow temperature range near the Curie point $\mathrm{T}_{C} \sim 170 \mathrm{~K}$, and it gradually changes to a semiconductive one below $150 \mathrm{~K}$. An external magnetic field strongly extends the metallic like temperature range above the Curie point; however, at low temperatures, the semiconductive behavior does not essentially change. Fig. 9 shows $\Delta \rho / \rho_{0}=[\rho(H)-\rho(H=0)] / \rho(H=0)$ as a function of a field at various temperatures for $\mathrm{La}_{0.5} \mathrm{Ba}_{0.5} \mathrm{CoO}_{3-\delta}$ cooled at $100^{\circ} \mathrm{C} / \mathrm{h}$. The magnetoresistance exhibits a local maximum near the $\mathrm{T}_{C}$ and increases gradually with further cooling. At $5 \mathrm{~K}$, we obtained the magnetoresistance (MR) value of about $20 \%$ in the field of $14 \mathrm{~T}$. The MR varies gradually with the field and does not show any tendency to saturation with the temperature decrease.

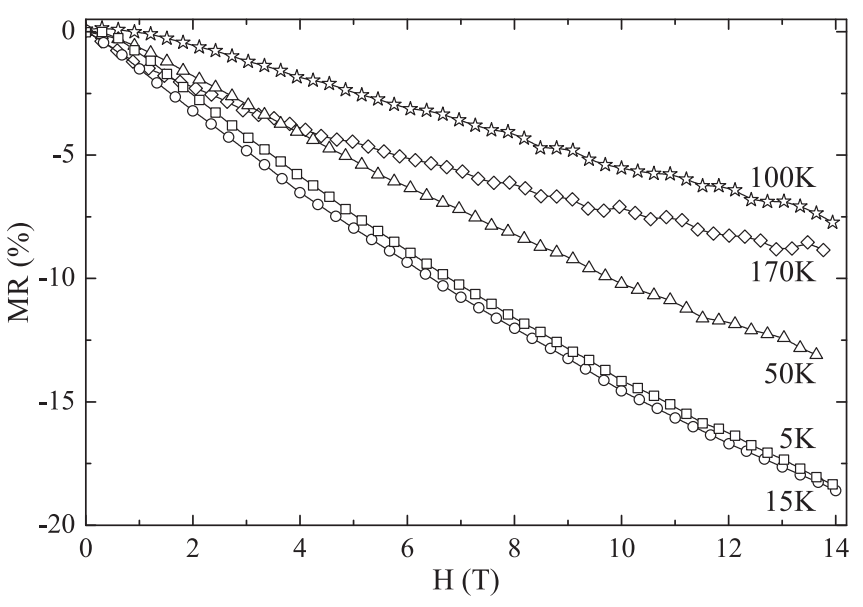

FIG. 9. Field dependencies of the magnetoresistance ratio $M R=[\rho(H)$ $-\rho(H=0)] / \rho(H=0) \cdot 100 \%$ for the ferromagnetic $\mathrm{La}_{0.5} \mathrm{Ba}_{0.5} \mathrm{CoO}_{3-\delta}$.

There are three different types of the large magnetoresistance ratio observed in the cobaltites. The large magnetoresistance was observed in the insulating spin glass phase of the lightly-doped $\mathrm{La}_{1-\mathrm{x}} \mathrm{Sr}_{\mathrm{x}} \mathrm{CoO}_{3}$ cobaltites. ${ }^{36,37}$ It is suggested that the intergranular effects associated with different magnetic and conductive states of ferromagnetic clusters and paramagnetic matrix are responsible for non-saturated magnetoresistance observed in the large magnetic field. ${ }^{36}$ This type of magnetoresistance is the most pronounced at low temperature. The second type of large magnetoresistance was observed at the low temperature in the insulating homogeneous ferromagnet $\mathrm{La}_{0.5} \mathrm{Ba}_{0.5} \mathrm{CoO}_{3} \cdot{ }^{10,11}$ This type of magnetoresistance has similar temperature and field dependencies with magnetotransport parameters of the lightly doped cobaltites. The magnetoresistance is not saturated in the large magnetic field and strongly increases while temperature decreases. The third type of magnetoresistance was observed in $\mathrm{LnBaCO}_{2} \mathrm{O}_{5.5}$ layered cobaltites and associated with the field-induced antiferromagnet-"ferromagnet" transition. $^{20,21}$ The magnetoresistance is saturated since antiferromagnet-"ferromagnet" transition is completed. All the three types of the magnetoresistance effects were observed only in the insulating phase of the cobaltites and had comparable magnitudes. In contrast to manganites, there is no large magnetotransport anomaly in the vicinity of the Curie point despite a different character of conductivity (conductive or insulating). The observation of the homogeneous ferromagnetism in insulating $\mathrm{La}_{0.5} \mathrm{Ba}_{0.5} \mathrm{CoO}_{3}$ in our opinion confirms the hypothesis that ferromagnetic properties of the hole-doped cobaltites with the perovskite structure are governed by the superexchange interactions similar to those in oxides of the non-mixed valence type of magnetoactive ions (e.g., ferrites with $\mathrm{Fe}^{3+}$ ions). The small magnetotransport and conductivity anomalies near the Curie point of the both insulating and conductive cobaltites apparently do not support the dominant role of the "double exchange" in defining of the ferromagnetic ordering as it was suggested in a number of works. ${ }^{6,10,11}$ The insulating character of the $\mathrm{La}_{0.5} \mathrm{Ba}_{0.5} \mathrm{CoO}_{3}$ ferromagnetic phase cannot be understood by means of the "itinerant magnetism" model. Apparently large magnetoresistance observed in homogeneous 
$\mathrm{La}_{0.5} \mathrm{Ba}_{0.5} \mathrm{CoO}_{3}$ at low temperature is associated with decreasing of the insulating gap between $\mathrm{e}_{g}$ and collective $\mathrm{t}_{2 g}$ state upon external magnetic field.

\section{CONCLUSIONS}

We have presented a detailed set of structural, magnetization, and magnetotransport measurements on polycrystalline $\mathrm{La}_{0.5-\mathrm{x}} \mathrm{Pr}_{\mathrm{x}} \mathrm{Ba}_{0.5} \mathrm{CoO}_{3-\delta}$ samples in the composition range $(0 \leq \mathrm{x} \leq 0.375 ; \delta<0.25)$. The increase of the Pr content leads to a transition from the cubic $(P m \overline{3} m)$ to tetragonal ( $P 4 /$ $\mathrm{mmm}$ ) structure due to ordering of the rare-earth and barium ions. In the both cubic and tetragonal phases, the oxygen vacancies are distributed randomly over the lattice. The decrease of the oxygen content leads to a transformation of the ferromagnetic structure into the G-type antiferromagnetic one via the intermediate region of the compositions demonstrating a macroscopic phase separation into different magnetic phases. The lowering temperature leads to unusual expansion of the lattice apparently associated with stabilization of the $\mathrm{Co}^{3+}$ ions in the high/low-spin state. The compositions with dominant G-type antiferromagnetic component show maximum of the magnetization. It is assumed that the maximum of the magnetization can be a result of the interaction between ferromagnetic clusters and antiferromagnetic phase as well as spin state transition. The ferromagnetic phase in the magnetically ordered state exhibits semiconductor-like behavior of the resistivity and a relatively large magnetoresistance at low temperature. The nonmetallic character of the ferromagnetic phase as well as weakness of magnetotransport anomalies near the Curie point probably indicate the important role of superexchange interactions via the intermediate oxygen ion in a stabilization of the ferromagnetic phase in the cobaltites with perovskite structure.

\section{ACKNOWLEDGMENTS}

The authors would like to acknowledge the financial support of the BRFFI (Grant No. T11D-003) and the FCT (Grant No. SFRH/BPD/42506/2007). This research project has also been partly supported by the European Commission under the Seventh Framework Programme through the Key Action: Strengthening the European Research Area, Research Infrastructures [Contract No. 226507 (NMI3)].

${ }^{1}$ J. B. Goodenough and J. S. Zhou, Structure and Bonding (Springer, New York, 2001), pp. 17-113.

${ }^{2}$ A. Podlesnyak, S. Streule, J. Mesot, M. Medarde, E. Pomjakushina, K. Conder, A. Tanaka, M. W. Haverkort, and D. I. Khomskii, Phys. Rev. Lett. 97, 247208 (2006)

${ }^{3}$ M. W. Haverkort, Z. Hu, J. C. Cezar, T. Burnus, H. Hartmann, M. Reuther, C. Zobel, T. Lorenz, A. Tanaka, N. B. Brookes, H. H. Hsieh, H. J. Lin, C. T. Chen, and L. H. Tjeng, Phys. Rev. Lett. 97, 176405 (2006).

${ }^{4}$ D. P. Kozlenko, N. O. Golosova, Z. Jirak, L. S. Dubrovinsky, B. N. Savenko, M. G. Tucker, Y. LeGodec, and V. P. Glazkov, Phys. Rev. B 75, 064422 (2007).
${ }^{5}$ R. F. Klie, J. C. Zheng, Y. Zhu, M. Varela, J. Wu, and C. Leighton, Phys. Rev. Lett. 99, 047203 (2007).

${ }^{6}$ J. Wu and C. Leighton, Phys. Rev. B 67, 174408 (2003).

${ }^{7}$ P. Tong, J. Yu, Q. Huang, K. Yamada, and D. Louca, Phys. Rev. Lett. 106, 156407 (2011).

${ }^{8}$ M. Kriener, C. Zobel, A. Reichl, J. Baier, M. Cwik, K. Berggold, H. Kierspel, O. Zabara, A. Freimuth, and T. Lorenz, Phys. Rev. B 69, 094417 (2004).

${ }^{9}$ A. P. Sazonov, I. O. Troyanchuk, H. Gamari-Seale, V. V. Sikolenko, K. L. Stefanopoulos, G. K. Nikolaides, and Y. K. Atanassova, J. Phys.: Condens. Matter 21, 156004 (2009).

${ }^{10}$ F. Fauth, E. Suard, and V. Caignaert, Phys. Rev. B 65, 060401(R) (2001).

${ }^{11}$ T. Nakajima, M. Ichihara, and Y. Ueda, J. Phys. Soc. Jpn. 75, 1572 (2005).

${ }^{12}$ V. Sikolenko, A. Sazonov, I. Troyanchuk, D. Többens, U. Zimmermann, E. Pomjakushina, and H. Szymczak, J. Phys.: Condens. Matter 16, 7317 (2004).

${ }^{13}$ V. Sikolenko, V. Efimov, E. Efimova, A. Sazonov, C. Ritter, A. Kuzmin, and I. Troyanchuk, J. Phys.: Condens. Matter 21, 436002 (2009).

${ }^{14}$ N. Sundaram, Y. Jiang, I. E. Anderson, D. P. Belanger, C. H. Booth, F. Bridges, J. F. Mitchell, T. Proffen, and H. Zheng, Phys. Rev. Lett. 102, 026401 (2009).

${ }^{15}$ J. B. Goodenough, Mater. Res. Bull. 6, 967 (1971).

${ }^{16}$ M. Senaris-Rodriguez and J. Goodenough, J. Solid State Chem. 118, 323 (1995).

${ }^{17}$ D. Louca, J. L. Sarrao, J. D. Thompson, H. Roder, and G. H. Kwei, Phys. Rev. B 60, 10378 (1999).

${ }^{18}$ I. O. Troyanchuk, D. V. Karpinsky, M. V. Bushinsky, V. Sikolenko, V. Efimov, and A. Cervellino, Pis'ma v Zh. Eksp. Teor. Fiz. 93, 149 (2011).

${ }^{19}$ R. P. Haggerty and R. Seshadri, J. Phys.: Condens. Matter 16, 6477 (2004).

${ }^{20}$ A. Maignan, C. Martin, D. Pelloquin, N. Nquyen, and B. Raveau, J. Solid State Chem. 142, 247 (1999).

${ }^{21}$ I. O. Troyanchuk, N. V. Kasper, D. D. Khalyavin, H. Szymczak, R. Szymczak, and M. Baran, Phys. Rev. Lett. 80, 3380 (1998).

${ }^{22}$ M. Soda, Y. Yasui, M. Ito, S. Iikubo, M. Sato, and K. Kakurai, J. Phys. Soc. Jpn. 73, 464 (2004).

${ }^{23}$ E. L. Rautama, V. Caignaert, P. Boullay, A. K. Kundu, V. Pralong, M. Karppinen, C. Ritter, and B. Raveau, Chem. Mater. 21, 102 (2009).

${ }^{24}$ V. P. Plakhty, Y. P. Chernenkov, S. N. Barilo, A. Podlesnyak, E. Pomjakushina, E. V. Moskvin, and S. V. Gavrilov, Phys. Rev. B 71, 214407 (2005).

${ }^{25}$ D. D. Khalyavin, D. N. Argyriou, U. Amann, A. A. Yaremchenko, and V. V. Kharton, Phys. Rev. B 75, 134407 (2007).

${ }^{26}$ F. Fauth, E. Suard, V. Caignaert, and I. Mirebeau, Phys. Rev. B 66, 184421 (2002).

${ }^{27}$ M. Soda, Y. Yasui, T. Fujita, T. Miyashita, M. Sato, and K. Kakurai, J. Phys. Soc. Jpn. 72, 1729 (2003).

${ }^{28}$ A. A. Taskin, A. N. Lavrov, and Y. Ando, Phys. Rev. Lett. 90, 227201 (2003).

${ }^{29}$ D. D. Khalyavin, I. O. Troyanchuk, N. V. Kasper, Q. Huang, J. W. Lynn, and H. Szymczak, J. Mater. Res. 17, 838 (2002).

${ }^{30}$ M. Soda, Y. Yasui, M. Ito, S. Iikubo, M. Sato, and K. Kakurai, J. Phys. Soc. Jpn. 73, 2857 (2004).

${ }^{31}$ M. Soda, Y. Yasui, Y. Kobayashi, T. Fujita, M. Sato, and K. Kakurai, J. Phys. Soc. Jpn. 75, 104708 (2006).

${ }^{32}$ I. O. Troyanchuk, D. V. Karpinsky, and F. Yokaichiya, J. Phys.: Condens. Matter 20, 335228 (2008).

${ }^{33}$ I. O. Troyanchuk, M. V. Bushinsky, V. M. Dobryanskii, and N. V. Pushkarev, JETP Lett. 94, 849 (2011).

${ }^{34}$ J. Rodriguez-Carvajal, Physica B 192, 55 (1993).

${ }^{35}$ G. Aurelio, J. Curiale, R. D. Sánchez, and G. J. Cuello, J. Phys.: Condens. Matter 21, 326002 (2009).

${ }^{36}$ J. Wu, J. W. Lynn, C. J. Glinka, J. Burley, H. Zheng, J. F. Mitchell, and C. Leighton, Phys. Rev. Lett. 94, 037201 (2005).

${ }^{37}$ M. Sánchez-Andújar, J. Mira, J. Rivas, and M. A. Señarís-Rodríguez, Prog. Solid State Chem. 35, 407 (2007). 